\title{
Lipatov's effective action, color glass condensate and classical gluon field of relativistic color charge
}

\author{
S.Bondarenko* \\ Physics Department, Ariel University, Israel \\ E-mail: sergeybeariel.ac.il
}

We discuss application of formalism of small- $x$ effective action for reggeized gluons (Lipatov's effective action), $[1,2,3]$, for the calculation of classical gluon field of relativistic color charge. The equations of motion with the reggeon fields are solved in LO and NLO approximations and the LO results are compared to the calculations performed in the CGC framework. It is demonstrated that the CGC results for the classical field are reproduced in our calculations.

XXV International Workshop on Deep-Inelastic Scattering and Related Subjects 3-7 April 2017

University of Birmingham, UK

\footnotetext{
* Speaker.
} 


\section{Introduction}

In the framework of perturbative QCD, the calculations of classical field created by a relativistic color charge is an important task. In the context of the Color Glass Condensate (CGC) approach, the knowledge of solution of the equations of motion is an initial set up for the further small- $x$ evolution of the gluonic system, see $[4,5]$. The Balitsky, Fadin, Kuraev, Lipatov (BFKL) like, [3], small- $x$ behavior of the gluon density, a non-linear Gribov, Levin, Ryskin (GLR) equation, see [6] as well as Balitsky-Kovchegov (BK) like, [7], corrections to this density were reproduced in the framework of this approach. The effective action approach of [2], in turn, can be considered as some generalization of Gribov's Regge calculus, [1], for the case of QCD degrees of freedom. Besides the usual gluon field, it includes two additional reggeon fields and widely used for the calculations of different quasi-elastic LO and NLO production amplitudes in the multi-Regge kinematics, see [8], or calculation of NLO corrections to the BKP, [9], kernel, see [10].

In our calculations, we use the effective action for reggeized gluons exploring ideas of [2]. Using light-cone gauge, we consider a problem with only one longitudinal gluon field in the equations of motion included. With the two reggeon fields present in the approach, the first reggeon field is defined as a LO value of the corresponding gluon field, whereas the second reggeon field arises as a source term in the Lagrangian.

\section{Effective action for reggeized gluons with color field source}

The effective action, see [2], is a non-linear gauge invariant action which correctly reproduces the production of the particles in direct channels at a quasi-multi-Regge kinematics:

$$
S_{\text {eff }}=-\int d^{4} x\left(\frac{1}{4} G_{\mu v}^{a} G_{a}^{\mu v}+\operatorname{tr}\left[\left(A_{+}\left(v_{+}\right)-A_{+}\right) j_{\text {reg }}^{+}+\left(A_{-}\left(v_{-}\right)-A_{-}\right) j_{\text {reg }}^{-}\right]\right),
$$

where

$$
A_{ \pm}\left(v_{ \pm}\right)=\frac{1}{g} \partial_{ \pm} O\left(x^{ \pm}, v_{ \pm}\right)=v_{ \pm} O\left(x^{ \pm}, v_{ \pm}\right),
$$

with $O\left(x^{ \pm}, v_{ \pm}\right)$as some operators, see further. The form of reggeon current we take is the following:

$$
j_{\text {rega }}^{ \pm}=\frac{1}{C(R)} \partial_{i}^{2} A_{a}^{ \pm},
$$

where $C(R)$ is the eigenvalue of Casimir operator in the representation $\mathrm{R}, C(R)=N$ in the case of adjoint representation used in the paper. There are additional kinematical constraints for the reggeon fields

$$
\partial_{-} A_{+}=\partial_{+} A_{-}=0
$$

corresponding to the strong-ordering Sudakov components in the multi-Regge kinematics, see [2]. We can rewrite Eq. (2.1) as

$$
S_{e f f}=-\int d^{4} x\left(\frac{1}{4} G_{\mu \nu}^{a} G_{a}^{\mu v}+v_{-} J^{-}\left(v_{-}\right)+v_{+} J^{+}\left(v_{+}\right)\right)
$$

with

$$
J^{ \pm}\left(v_{ \pm}\right)=O\left(v_{ \pm}\right) j_{r e g}^{ \pm}
$$


Under variation on the gluon fields these currents reproduce the Lipatov's induced currents, see Appendix:

$$
\delta\left(v_{ \pm} J^{ \pm}\left(v_{ \pm}\right)\right)=\left(\delta v_{ \pm}\right) j_{\mp}^{\text {ind }}\left(v_{ \pm}\right)=\left(\delta v_{ \pm}\right) j^{ \pm}\left(v_{ \pm}\right),
$$

which posseses a covariant conservation property:

$$
\left(D_{ \pm} j_{\mp}^{\text {ind }}\left(v_{ \pm}\right)\right)^{a}=\left(D_{ \pm} j^{ \pm}\left(v_{ \pm}\right)\right)^{a}=0 .
$$

Here and further we denote the induced current in the component form in the adjoint representation as

$$
j_{a}^{ \pm}\left(v_{ \pm}\right)=-\operatorname{ltr}\left[T_{a} j^{ \pm}\left(v_{ \pm}\right)\right]=\frac{1}{N} \operatorname{tr}\left[f_{a} O f_{b} O^{T}\right]\left(\partial_{i}^{2} A_{-}^{b}\right) .
$$

\section{The LO solution and LO structure of the effective action}

At the presence of an external source the solution of the equations of motion can be presented in the form of the following ansatz for the gluon fields:

$$
v_{-}=0, v_{\perp}=\Lambda\left[g A_{+}\right]+g \Lambda_{1}\left[g A_{+}\right]+\cdots, v_{+}=A_{+}+g \Phi\left[g A_{+}, v_{\perp}\right]+\cdots, \partial_{-} A_{+}=0 .
$$

The calculations give to LO accuracy:

$$
v_{a+}=A_{a+}
$$

see [2] and

$$
v_{i}^{b}\left(x^{+}, x_{\perp}\right)=U^{b c}\left(A_{+}\right) \rho_{c i}\left(x^{-}, x_{\perp}\right) .
$$

Here the form of $U^{a b}$ function in Eq. (3.3) can be written consistently with the used in [5]:

$$
v_{i}^{b}=\operatorname{tr}\left[f^{b} U_{-\infty, x^{+}}\left(v_{+}\right) f^{c} U_{x^{+}, \infty}\left(v_{+}\right)\right] \rho_{c i}\left(x^{-}, x_{\perp}\right)=U^{b c} \rho_{c i}\left(x^{-}, x_{\perp}\right),
$$

with $U_{-\infty, x^{+}}=P e^{g \int_{-\infty}^{x^{+}} d x^{\prime}{ }^{+} V_{+}^{a}}$, which correspond to the form of the induced current in the effective action when $O$ operator is taken in the form of simple ordered exponential. The form of the function $\rho_{c i}\left(x^{-}, x_{\perp}\right)$ is arbitrary in this case, it is proportional to the color charge density in the CGC approach. In the case of effective action for reggeized gluons approach, we consider solution of equations of motion as solution for the classical gluon field in the presence of $A_{-}$source, i.e. the following substitution exists:

or

$$
\partial_{i} \partial_{-} \rho_{a}^{i}=-\frac{1}{N} \partial_{\perp}^{2} A_{a}^{+}
$$

$$
\rho_{a}^{i}=\frac{1}{N} \partial_{-}^{-1}\left(\partial^{i} A_{-}^{a}\right)
$$

Correspondingly, we obtain for Eq. (3.4):

$$
v_{i}^{a}=\frac{1}{N} U^{a b}\left(v_{+}\right)\left(\partial_{-}^{-1}\left(\partial_{i} A_{-}^{b}\right)\right) .
$$

Inserting obtained classical gluon fields solutions in the Eq. (2.1) action, we will obtain a action which will depend only on the reggeon fields, see [2], determining the LO RFT action of the approach:

$$
S_{e f f}=-\int d^{4} x\left(s_{1}\left[g, A_{+}, A_{-}\right]+g s_{2}\left[g, A_{+}, A_{-}\right]+\cdots\right) .
$$


With LO accuracy we have:

$$
S_{e f f}=-\int d^{4} x\left(\left(\partial_{i} A_{+}^{a}\right) U^{a b}\left(A_{+}\right)\left(\partial_{-} \rho_{i b}\left(x_{\perp}\right)\right)+\frac{1}{N} A_{+}^{a}\left(O^{a b}\left(A_{+}\right)+N \delta^{a b}\right)\left(\partial_{\perp}^{2} A_{-}^{b}\right)\right),
$$

with $O^{a b}\left(A_{+}\right)=\operatorname{Tr}\left[f^{a} O\left(A_{+}\right) f^{b}\right]$ in adjoint representaion. This expression can be also rewritten as

$$
S_{\text {eff }}=-\frac{1}{N} \int d^{4} x\left(\left(\partial_{i} A_{+}^{a}\right) U^{a b}\left(A_{+}\right)\left(\partial_{i} A_{-}^{b}\right)+A_{+}^{a}\left(O^{a b}\left(A_{+}\right)+N \delta^{a b}\right)\left(\partial_{\perp}^{2} A_{-}^{b}\right)\right) .
$$

We obtained, that due the ordered exponential in the action, there are some additional corrections which were not considered in [2]. However, obtained in expression Eq. (3.10) corrections are not complete, we also need the higher order solutions of equations of motion.

\section{Conclusion}

In this paper we consider application of the effective action approach for reggeized gluons to the calculation of a classical gluon field produced by relativistic color charge. We demonstrate, that effective action for reggeized gluons can be obtained from QCD action, when both reggeon fields are introduced as non-zero LO solutions for the classical longitudinal gluon fields and as sources of each other. We also obtained, that LO expressions for the classical gluon fields calculated in the effective action formalism, reproduce results of CGC approach. Another result of the paper, is that we calculated classical gluon field in the presence of external reggeon fields to NLO accuracy in the light-cone gauge, see [2]. We also note, that proposed formalism is very important for the construction of QCD based RFT calculus.

\section{Appendix A: Induced current in the effective action}

In this Appendix we consider an $j^{+}$component of the induced current which can be obtained by variation of the current term in the effective action Eq. (2.1):

$$
j_{\text {ind }}^{+}\left(v_{+}\right)=j_{-}^{i n d}\left(v_{+}\right)=\frac{1}{N} O\left(v_{+}\right)\left(\partial_{i}^{2} A^{+}\right) O^{T}\left(v_{+}\right) .
$$

The operators $O$ and $O^{T}$ are introduced in [2]:

$$
O=\partial_{+}\left(D_{+}^{-1}\right) ; O^{T}=\left(D_{+}^{-1}\right) \overleftarrow{\partial}_{+}
$$

and have the following properties:

$$
\partial_{+} O=g V_{+} O, O^{T} \overleftarrow{\partial}_{+}=-g O^{T} v_{+},
$$

see Appendix C further. The Eq. (A.1) form of the current is general, the particular representations of the current in terms of P-exponentials, in turn, depend on the representations of $O$ operator, or, more precisely on the representation of the $\partial_{+}^{-1}$ operator. If we take the following simplest representation

$$
\frac{1}{\partial_{+}} f\left(x^{+}\right)=\int_{-\infty}^{x^{+}} d x^{\prime} f\left(x^{\prime}\right)
$$


we obtain for these operators:

$$
O=P e^{l g \int_{-\infty}^{x^{+}} d x^{\prime}+V_{+}^{a} T_{a}}
$$

and

$$
O^{T}=P e^{\imath g \int_{x^{+}}^{\infty} d x^{\prime}+V_{+}^{a} T_{a}} .
$$

The variation of interaction term in the action can be calculated and results by induced current from Eq. (A.1):

$\delta\left(A_{+}\left(v_{+}\right) j_{\text {reg }}^{+}\right)=-\imath\left(\delta v_{+}^{a}\right) \operatorname{tr}\left[T_{a} j_{\text {ind }}^{+}\left(v_{+}\right)\right]=\left(\delta v_{+}^{a}\right) j_{a}^{+}\left(v_{+}\right)=-\frac{1}{N}\left(\delta v_{+}^{a}\right) \operatorname{tr}\left[T_{a} O T_{b} O^{T}\right]\left(\partial_{i}^{2} A_{b}^{+}\right)$,

with $v_{+}=\imath T^{a} v_{+}^{a}$ representation of the gluon field used. In the case of adjoint representation ${ }^{1}$ we will obtain:

$\delta\left(A_{+}\left(v_{+}\right) j_{r e g}^{+}\right)=\left(\delta v_{+}^{a}\right) j_{a}^{+}\left(v_{+}\right)=\frac{1}{N}\left(\delta v_{+}^{a}\right) \operatorname{tr}\left[f_{a} O f_{b} O^{T}\right]\left(\partial_{i}^{2} A_{b}^{+}\right)=\frac{1}{N}\left(\delta v_{+}^{a}\right) U^{a b}\left(\partial_{i}^{2} A_{b}^{+}\right)$,

that provides

$$
j_{a}^{+}\left(v_{+}=0\right)=-\partial_{i}^{2} A_{a}^{+} .
$$

The $U^{a b}$ exponential in Eq. (A.8) is the same as used in QGC approach of [5]. The Eq. (A.5)Eq. (A.6) forms of the operators can be modified in order to provide the action's unitarity at $x \rightarrow$ $\pm \infty$. For that, the Eq. (A.4) operator can be modified as ${ }^{2}$ :

$$
\frac{1}{\partial_{+}} f\left(x^{+}\right)=\frac{1}{2} \int d x^{\prime} \varepsilon\left(x^{+}-x^{\prime}\right) f\left(x^{\prime}\right),
$$

where $\varepsilon\left(x^{+}-x^{\prime}\right)$ is a sign function, that corresponds to the symmetrical, in the sense of Eq. (A.5)Eq. (A.6) expressions, ordered exponentials in the presentation of the operator and principal value prescription of regularization of corresponding $1 / k_{+}$pole in momentum space, see details in [2]. In this case, more complicated expressions for the operators will be obtained.

\section{References}

[1] V. N. Gribov, Sov. Phys. JETP 26 (1968) 414.

[2] L. N. Lipatov, Nucl. Phys. B 452, 369 (1995); Phys. Rept. 286, 131 (1997); Subnucl. Ser. 49, 131 (2013); Int. J. Mod. Phys. Conf. Ser. 39, 1560082 (2015); Int. J. Mod. Phys. A 31, no. 28/29, 1645011 (2016); EPJ Web Conf. 125, 01010 (2016).

[3] L. N. Lipatov, Sov. J. Nucl. Phys. 23, 338 (1976) [Yad. Fiz. 23 (1976) 642]; E. A. Kuraev, L. N. Lipatov and V. S. Fadin, Sov. Phys. JETP 45, 199 (1977) [Zh. Eksp. Teor. Fiz. 72, 377 (1977)]; I. I. Balitsky and L. N. Lipatov, Sov. J. Nucl. Phys. 28, 822 (1978) [Yad. Fiz. 28, 1597 (1978)].

[4] L. McLerran and R. Venugopalan, Phys. Rev. D49 (1994), 2233; D49 (1994), 3352.

\footnotetext{
${ }^{1}$ The general form of the current does not depend on the representation, in our particular case we take $\left(T_{a}\right)_{b c}=$ $-\imath f_{a b c}$ in the current, representing only "external" indexes in the expression.

${ }^{2}$ We use the Kogut-Soper convention for the metric tensor, in the Lepage-Brodsky convention there is $1 / 4$ coefficient in the front of the following expression.
} 
[5] J. Jalilian-Marian, A. Kovner, L. McLerran and H. Weigert, Phys.Rev. D55, 5414 (1997);

J. Jalilian-Marian, A. Kovner, A. Leonidov and H. Weigert, Nucl. Phys. B 504, 415 (1997);

J. Jalilian-Marian, A. Kovner, A. Leonidov and H. Weigert, Phys. Rev. D 59, 014014 (1998);

J. Jalilian-Marian, A. Kovner and H. Weigert, Phys. Rev. D 59, 014015 (1998); E. Iancu, A. Leonidov and L. D. McLerran, Nucl. Phys. A 692, 583 (2001); E. Iancu, A. Leonidov and L. D. McLerran, Phys. Lett. B 510, 133 (2001); E. Ferreiro, E. Iancu, A. Leonidov and L. McLerran, Nucl. Phys. A 703, 489 (2002).

[6] L. V. Gribov, E. M. Levin and M. G. Ryskin, Phys.Rep. 100, 1 (1983).

[7] I. Balitsky, Nucl. Phys. B463 (1996) 99; Y. V. Kovchegov, Phys. Rev. D60 (1999) 034008; Phys. Rev. D61 (2000) 074018.

[8] L. N. Lipatov, Nucl. Phys. Proc. Suppl. 99A, 175 (2001); M. A. Braun and M. I. Vyazovsky, Eur. Phys. J. C 51, 103 (2007); M. A. Braun, M. Y. Salykin and M. I. Vyazovsky, Eur. Phys. J. C 65, 385 (2010); M. A. Braun, L. N. Lipatov, M. Y. Salykin and M. I. Vyazovsky, Eur. Phys. J. C 71, 1639 (2011); M. A. Braun, M. Y. Salykin and M. I. Vyazovsky, Eur. Phys. J. C 72, 1864 (2012); M. Hentschinski and A. Sabio Vera, Phys. Rev. D 85, 056006 (2012); M. A. Braun, M. Y. Salykin, S. S. Pozdnyakov and M. I. Vyazovsky, Eur. Phys. J. C 72, 2223 (2012); J. Bartels, L. N. Lipatov and G. P. Vacca, Phys. Rev. D 86, 105045 (2012); M. A. Braun, S. S. Pozdnyakov, M. Y. Salykin and M. I. Vyazovsky, Eur. Phys. J. C 73, no. 9, 2572 (2013); G. Chachamis, M. Hentschinski, J. D. Madrigal Martinez and A. Sabio Vera, Phys. Part. Nucl. 45, no. 4, 788 (2014).

[9] J. Bartels, Nucl. Phys. B 175 (1980) 365; J. Kwiecinski, M. Praszalowicz, Phys. Lett. B 94 (1980) 413.

[10] J. Bartels, V. S. Fadin, L. N. Lipatov and G. P. Vacca, Nucl. Phys. B 867, 827 (2013). 